\title{
1 Rational design and implementation of a chemically inducible
} 2 hetero-trimerization system

3

4 Helen D. Wu ${ }^{1,5}$, Masaki Kikuchi², Onur Dagliyan ${ }^{3}$, Adam K. Aragaki $^{4,5}$, Hideki Nakamura ${ }^{5,8}$,

5 Nikolay V. Dokholyan ${ }^{6,7}$, Takashi Umehara ${ }^{2, *}$, Takanari Inoue $^{1,4,5,{ }^{*}}$

6

$7{ }^{1}$ Department of Biomedical Engineering, Johns Hopkins University School of Medicine,

8 Baltimore, MD 21205

92 Laboratory for Epigenetics Drug Discovery, RIKEN Center for Biosystems Dynamics Research,

10 Yokohama, Kanagawa 230-0045, Japan

$11{ }^{3}$ Department of Neurobiology, Harvard Medical School, Boston, MA 02115

$12{ }^{4}$ Department of Pharmacology, Johns Hopkins University School of Medicine, Baltimore, MD

1321205

$14{ }^{5}$ Department of Cell Biology, Center for Cell Dynamics, Johns Hopkins University School of

15 Medicine, Baltimore, MD 21205

$16{ }^{6}$ Departments of Pharmacology and Biochemistry \& Molecular Biology, Penn State University

17 College of Medicine, Hershey, Pennsylvania.

$18{ }^{7}$ Departments of Chemistry and Biomedical Engineering, Pennsylvania State University,

19 University Park, Pennsylvania.

$20{ }^{8}$ Present address: Kyoto University Graduate School of Engineering, Department of Synthetic

21 Chemistry and Biological Chemistry, Katsura Int'tech Center, Graduate School of Engineering,

22 Kyoto University, Nishikyo-ku, Kyoto, 615-8530, Japan

$25{ }^{*}$ Correspondence:

26 Takashi Umehara takashi.umehara@riken.jp for Structural Analysis

27 Takanari Inoue jctinoue@jhmi.edu for General Correspondence 


\section{Abstract}

29 Chemically inducible dimerization (CID) uses a small molecule to induce binding of two different 30 proteins. CID tools exemplified by the FKBP/FRB/rapamycin system have been widely employed

31 to probe molecular events inside and outside cells. While various CID tools are available,

32 chemically inducible trimerization (CIT) has not been developed, due to inherent challenges in

33 designing or identifying a chemical that simultaneously binds three proteins with high affinity

34 and target specificity. Nevertheless, by introducing a third recruitable component, CIT could

35 enable versatile applications. Here, we devised the CIT by rationally splitting FRB and FKBP. Based

36 on cellular and structural datasets, select split pairs of FRB or FKBP underwent efficient

37 trimerization with full length FKBP or FRB, respectively, upon addition of rapamycin. We also

38 demonstrated its potential for cellular applications by rapidly inducing tri-organellar plasma

39 membrane-ER-mitochondria junctions, and by perturbing intended membrane lipids exclusively

40 at the plasma membrane-ER membrane contact sites. By conferring one additional condition to

41 what is achievable with CID, CIT expands the types of manipulation in single live cells, to address

42 cell biology questions otherwise intractable, and engineer cell functions for future synthetic

43 biology applications. 


\section{Introduction}

45 Chemically inducible dimerization (CID) uses a bifunctional small molecule to bring together two proteins with high affinity, specificity and fast kinetics ${ }^{1,2}$. CID systems such as the rapamycin-

47 dependent FKBP-FRB heterodimerization (Fig. 1a) can exert tight spatiotemporal control over local protein concentrations by oligomerization ${ }^{3}$ or by rapidly translocating a protein of interest to or from a desired site of action, 5; translocation-based and other CID-based strategies have enabled signal parsing of a given protein from the noise of other cell signaling pathways, leading

51 to discoveries in cell migration, endocytosis, transcription, and translation ${ }^{1,2,6}$. The seconds-to-

52 minutes timescale that CID operates at offers a further advantage - CID strategies are less

53 susceptible to genetic compensation that sometimes plague knockdown (KD) or knockout (KO)

54 methods. When used as a building block for rewiring circuits in a bottom-up approach, CID

55 systems have created fast, translation-independent synthetic logic gates in mammalian cells ${ }^{7}$.

56 Over the years, CID has become a widely-used, easily generalizable chemical biology tool with

57 promising clinical impact ${ }^{8}$.

While there have been hundreds of applications of CID, no inducible trimerization system has been developed to our knowledge. Examples of transient or stable trimerization in biology include trimeric $\mathrm{G}$ proteins ${ }^{9}$, inner ear epithelial cell junctions ${ }^{10}$, and MHC-antigen-TCR complexes governing T-cell selection ${ }^{11}$. Engineering chemically inducible trimerization (CIT) could address biologically relevant questions, and such a system would expand in general the palette of what is achievable with chemical biology.

64 In theory, the simplest CIT system should comprise of three unique protein components that can

65 be brought together by a tri-functional small molecule. However, trimerization would be difficult 66 to achieve, as recruiting an additional protein doubles the possible bound states of the small 67 molecule compared to dimerization (Supplementary Fig. 1). Efficient trimerization requires that the trimeric complex be the most favorable state over other less bound configurations. For CID,

69 the secret to its high efficiency over a large range of rapamycin concentrations is positive cooperativity: When one CID protein binds rapamycin, the resulting rapamycin-protein complex

71 binds the second protein with thousand-fold higher affinity than rapamycin alone ${ }^{12}$. This unique 
72 cooperativity is rapamycin-dependent, and mediated through electrostatic interactions at the

73 FKBP-rapamcycin-FRB interface ${ }^{13}$. Because engineering de novo proteins with appropriate small

74 molecule-dependent surface interactions would be highly challenging, we instead exploited the

75 special properties of the FKBP-FRB CID system to achieve trimerization.

76 We report the generation of a novel CIT system whose components comprise split FRB and FKBP

77 proteins (Fig. 1a). Determined with the aid of computational analysis, candidate split proteins

78 were characterized for their efficacy of trimerization in cells. Formation of the trimer in vitro was

79 assessed by X-ray crystallography. CIT was used to target cytosolic proteins to regions of close

80 inter-organelle membrane junctions and to induce tri-organellar ER-plasma membrane-

81 mitochondria contacts. Finally, we used CIT to locally alter phosphoinositide composition of ER-

82 plasma membrane junctions to affect local lipid homeostasis. Altogether, CIT has small

83 components that trimerize with fast kinetics in a rapamycin-inducible manner, expanding the

84 variety of perturbations possible in the chemical biology toolkit. 


\section{Results}

\section{Design of split FRB and FKBP}

87 We narrowed down candidate split site regions of FRB and FKBP by avoiding regions forming 88 secondary structures, evolutionarily-conserved amino acids or residues crucial for CID 89 functionality. We used FRB (residues 2021-2113 of human mTOR) with the T2098L mutation, 90 which makes FRB otherwise unstable unless bound to chemical ligands or FKBP ${ }^{14}$, an

91 advantageous trait for low recombination potential after protein splitting (Fig. 1b). In splitting 92 FRB, we avoided its four alpha helices, crucial residues for FRB-FKBP-rapamycin stabilization 93 (L2031, S2035, Y2038, F2039, T2098L, W2101, D2102, and Y2105) ${ }^{13}$, and regions of high 94 conservation in the 3 FRB loops (Fig. 1c and Supplementary Fig. 2a,b). We then applied the SPELL 95 algorithm ${ }^{15}$ to pinpoint candidate split sites in FRB through computational analysis. All the loops 96 selected for splitting (Fig. 1b,c and Supplementary Fig. 2a) show low evolutionary conservation 97 with Kullback-Leibler conservation scores less than 2 and have solvent accessible area values

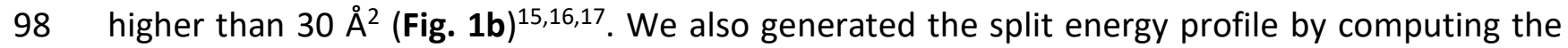
99 energy of the split parts relative to the native energy of the intact protein (Fig. 1 $\mathbf{b}$ ); the three 100 loops reside in two major energy wells, which suggest splitting at these sites would generate split 101 proteins that are less likely to pre-associate when co-expressed in the absence of rapamycin ${ }^{15}$. 102 Split sites for FKBP were also determined in this manner (Supplementary Fig. 4a,b). Hereafter, 103 split FRB or FKBP proteins are abbreviated as SFRB or sFKBP. Each split site is numbered in terms 104 of likelihood of success based on the computational analysis; sFRB $1, \mathrm{sFRB}_{2}$ and $\mathrm{sFRB}_{3}$ refer to 105 pairs of sFRB proteins split at sites 1, 2 or 3, with split site 1 most likely to succeed. The N- or C106 terminal half of a given $\mathrm{SFRB}$ protein pair is $\operatorname{sFRB} \chi_{N}$ or $\operatorname{sFRB} \chi_{c}, \chi$ being the split site number. $\mathrm{A}$ 107 similar naming convention was used for split FKBP.

108 To determine whether split proteins can be stably expressed, we generated plasmids of $\mathrm{N}$ 109 terminal fluorophore-tagged SFRB proteins and observed cytosolic expression in HeLa cells 110 (Supplementary Fig. 2c). In some cases, split proteins show ER accumulation, which may be 111 attributed to instability in protein folding. We also generated a series of Lyn or CAAX membrane112 tagged SFRB fusion proteins, all of which localized to the plasma membrane (Fig. 1d). To 
113 determine whether there is appreciable pre-association between sFRB pairs, we used the Lyn 114 motif to target sFRB $\chi_{N}$ to the plasma membrane (PM), observed the localization of corresponding

115 cytosolic sFRB $\chi$ c across all three split sites $(\chi=1,2,3)$, and determined PM/cytosol signal ratio 116 of the cytosolic protein (Fig. 1e,f). Higher PM/cytosol ratios occurred between Lyn-Y-sFRB $1 \mathrm{~N}$ and 117 CFP-sFRB 1 c compared to Lyn-Y-sFRB 1 and CFP alone $(0.83 \pm 0.01$ vs. $0.75 \pm 0.01, p<0.0001)$. In 118 contrast, cytosolic CFP-sFRB $2 \mathrm{~N}$ and $\mathrm{SFRB}_{3 \mathrm{~N}} \mathrm{PM} / \mathrm{cytosol}$ ratios did not differ from CFP alone $(0.80$ $119 \pm 0.01$ vs. $0.77 \pm 0.01,0.82 \pm 0.01$ vs. $0.84 \pm 0.01$, respectively) with the same experimental setup, 120 suggesting the $\mathrm{SFRB}_{1}$ pair has higher tendency to pre-associate. This approach to designing split 121 sites, validating cytosolic protein expression, and generating membrane-anchored protein was 122 also applied to sFKBP.

\section{Characterization of split FRB}

124 The sFRB protein pairs were tested for their ability to bind FKBP in a rapamycin-inducible manner 125 to achieve CIT. We co-expressed cytosolic FKBP with PM-localized sFRB pairs in HeLa cells. If 126 functional, rapamycin-induced FKBP binding to sFRB pairs would shift FKBP localization from the 127 cytosol to the PM (Fig. 2a).sFRB pairs were localized on the PM with Lyn and CAAX tags (Fig. 1d). 128 Upon rapamycin addition, all three sFRB pairs indeed recruited cytosolic mCh-FKBP to the PM, 129 comparable to FKBP recruitment by full length (FL) FRB (Fig. 2b). Changes in cell linescans reflect 130 the cytosol-to-PM localization shift pre- and 9 mins post-rapamycin administration (Fig. 2b). 131 Percent change in mCh-FKBP PM/cytosol ratios derived from linescans was positive for all sFRB 132 pairs tested; values were not significantly different between $\mathrm{SFRB}_{1}, \mathrm{SFRB}_{2}$, and $\mathrm{sFRB}$, versus full 133 length FRB ( $\mathbf{p}=\mathbf{0 . 6 1 3}, \mathbf{0 . 0 5 7}, \mathbf{0 . 5 7 9}$ respectively, Fig. $\mathbf{2 c})$. However, no sFRB ${ }_{c}$ half could recruit 134 FKBP to the PM without co-expression of its cognate $s F R B_{N}$ half and vice versa $(p<0.0001$ for all 135 cases, Fig. 2c). These results suggest that PM-localized sFRB halves are as efficient as full length 136 FRB in recruiting FKBP in the presence of rapamycin, but each sFRB half cannot independently 137 recruit FKBP. However, in the absence of FKBP overexpression, sFRB halves can still dimerize 138 (Supplementary Fig. 3), as shown by rapamycin-dependent recruitment of cytosolic sFRB $\chi_{c}$ to 139 PM-localized Lyn-YFP-sFRB $\chi_{N}(\chi=1,2,3)$. This is most likely due to endogenous FKBP-rapamycin 140 complexes in the cell ${ }^{18}$, and/or due to rapamycin alone bringing sFRB pairs together. sFKBP pairs 
141 were also screened, and 3 out of 4 split sites $(\chi=1,2,3)$ produced functional sFKBP CIT systems

142 (Fig. 3f and Supplementary Fig. 4).

143 The kinetics of cytosolic FKBP recruitment to the PM was determined from exponential

144 regression of cytosolic FKBP intensity decay during translocation. Cytosolic FKBP signal decay was 145 captured by confocal imaging acquisition at $10 \mathrm{~s}$ intervals. The rate constant of cytosolic FKBP 146 signal decay with the full-length FRB was significantly higher than with the sFRB 1 pair $(0.085 \pm$ $1470.0075 \mathrm{~s}^{-1}$ versus $0.058 \pm 0.0047 \mathrm{~s}^{-1}, \mathrm{p}=0.008$; Fig. $2 \mathrm{~d}$ ), indicating that $\mathrm{sFRB}$, has slower FKBP 148 recruitment kinetics upon rapamycin addition, although the timescale of FKBP recruitment by 149 the $\mathrm{SFRB}_{1}$ pair still falls within the minutes range. CID is practically irreversible in cells due to low $150 k_{\text {off, }}$, but introduction of split sites could affect $k_{\text {off }}$ of sFRB pairs. To test for irreversibility, cells 151 expressing PM-localized Lyn-YFP-sFRB $\chi_{N}$, cytosolic CFP-sFRB $\chi_{c}(\chi=1,2,3)$ and cytosolic mCh152 FKBP were incubated with rapamycin for 30 mins. Samples were then washed ten times, and cells 153 showing trimerization were imaged over 30 mins (Supplementary Fig. 5a). No appreciable 154 decrease in PM/cytosol ratio was detected for either CFP-sFRB $\chi$ or mCh-FKBP (Supplementary 155 Fig. 5b), indicating that SFRB CIT systems are practically irreversible. To investigate whether the 156 three sFRB pairs work in an orthogonal manner, we alternated $\mathrm{SFRB}_{\mathrm{N}}$ with $\mathrm{sFRB} \mathrm{C}_{\mathrm{C}}$ halves split at 157 different sites. While we determined that SFRB pairs are non-orthogonal, we discovered three 158 additional $s F R B$ pairs $\left(s F R B_{1 N} / s F R B_{3 C}, s F R B_{2 N} / s F R B_{1 C}, s F R B_{2 N} / s F R B_{3 C}\right)$ that trimerize with FKBP

159 (Supplementary Fig. 6).

\section{Structural validation of split FKBP}

161 First, we solved the crystal structure of a complex of unsplit FKBP12 and FRB (T2098L) proteins 162 with rapamycin to examine whether the point mutation of FRB affects the overall conformation 163 and/or the interaction with rapamycin. The crystal structure of FKBP12-rapamycin·FRB (T2098L) 164 determined at $2.20 \AA$ resolution (Fig. 3a) shows only $0.61 \AA$ of the average root-mean-square 165 deviation (RMSD) for $\mathrm{C} \alpha$ atoms to the wild-type FRB-containing complex (PDB ID: 1FAP), 166 indicating that the both structures are in almost perfect agreement. Also, the major interactions 167 between FRB (T2098L), FKBP12 and rapamycin were the same as in the wild-type 
FRB·FKBP12-rapamycin complex (Fig. 3b). Hence, we confirmed that the T2098L mutation of FRB does not affect the overall conformation and the interactions with rapamycin.

170 Next, we attempted to purify $\operatorname{sFRB} \chi$ and $\operatorname{sFKBP} \chi$ fragments and reconstitute their complexes to

171 structurally validate our split strategy. We could purify sFKBP ${ }_{1 \mathrm{~N}}$ as an MBP fusion protein by an

172 E. coli expression system although we could not obtain the $\mathrm{sFRB} \chi_{\mathrm{N}}$ fragments as a soluble protein.

173 Therefore, we aimed to structurally validate whether sFKBP $1 \mathrm{~N}$ and $\mathrm{SFKBP}_{1 \mathrm{c}}$ form a functional

$174 \mathrm{sFKBP}_{1}$ complex. We solved crystal structures of $\mathrm{sFKBP}{ }_{1} \cdot$ rapamycin and $\mathrm{sFKBP} \mathrm{P}_{1} \cdot$ rapamycin $\cdot \mathrm{FRB}$

175 (T2098L) at 2.92-3.11 A resolution (Fig. 3c,d). In both structures, sFKBP $1 \mathrm{~N}$ and sFKBP $1 \mathrm{c}$ formed

176 the same secondary structure as FKBP12, showing only 0.38 and $0.42 \AA$ of the average RMSD for

$177 \mathrm{C} \alpha$ atoms to the corresponding region of the intact FKBP12 protein, respectively. The interactions

178 between $\mathrm{SFKBP}_{1}$ and rapamycin are completely conserved in both structures. Finally, the overall

179 structure of $\mathrm{SFKBP}_{1} \cdot$ rapamycin·FRB (T2098L) shows only $0.79 \AA$ of the average RMSD to the intact

180 FKBP12-rapamycin·FRB (T2098L) complex, without disrupting hydrogen bonds and major

181 hydrophobic interactions to FRB and rapamycin (Fig. 3e). These results indicate that, in vitro, the

182 split FKBP fragments form a functional FKBP protein which is structurally indistinguishable to the

183 intact FKBP protein.

\section{CIT at organelle membrane junction sites}

185 Membrane contact sites (MCS), also called organelle-organelle junctions, are subcellular regions

186 of close inter-organellar membrane apposition where unique signaling can occur, allowing

187 organelles of specialized biochemical function to coordinate with each other while retaining their

188 distinct identities ${ }^{4,19,20}$. MCS are widespread among almost all membrane-bound organelles ${ }^{21,22}$,

189 and local MCS molecular tethers and actuators ${ }^{23}$ regulate processes ranging from calcium ${ }^{24,25,26}$

190 and lipid transport ${ }^{27}$ to organelle fission ${ }^{28,29}$. Functional studies for MCS are complex; most MCS

191 regulate multiple functions, and local MCS proteins play compensatory roles, such that few MCS

192 have been completely ablated by the knock-down or knock-out of a single gene ${ }^{30}$. These

193 confounding conditions make rapidly inducible perturbation attractive for studying MCS, as

194 supported by prior CID-based studies in the field ${ }^{4}$ 19, 20, 31. As proof-of-principle, we used CIT to 
target cytosolic protein to ER-mitochondria and ER-PM MCS, demonstrating its potential as a generalizable recruitment-based screen for MCS function (Fig. 4a).

197 To recruit cytosolic protein to ER-PM junctions, we co-expressed PM-targeted sFRB 1 (Lyn-CFP-

$198 \mathrm{sFRB}_{1 \mathrm{~N}}$ ) and ER-targeted sFRB $1 \mathrm{c}(\mathrm{YFP}-\mathrm{sFRB} 1 \mathrm{C}-\mathrm{Cb5}$ ) with cytosolic mCh-FKBP (Fig. 4a). Upon 199 rapamycin addition, ER and PM tethering formed patches that colocalize with mCh-FKBP (Fig. 4b

200 and Supplementary Video 1). Pairwise image correlation between all three image wavelengths

201 all significantly increased after rapamycin ( $p<0.0001$, Fig. 4d), suggesting that the CIT system can

202 recruit cytosolic protein to the ER-PM MCS. When we removed sFRB $1 \mathrm{~N}$ (using Lyn-CFP only) or 203 sFRB $_{1 C}$ (using only YFP-Cb5) from the experiment, trimerization could not be observed

204 (Supplementary Fig S7a,b, left and center) with no increase in image correlation coefficients 205 between all three signals. However, when mCh alone was expressed instead of mCh-FKBP, ER-

206 PM tethering still occurred, albeit with no mCh recruitment to the site, and the image correlation

207 coefficient between the ER and PM signals significantly increased (Supplementary Fig S7a,b, 208 right).

209 We also used sFKBP to recruit cytosolic FRB-mCh to ER-PM junctions. Cells showed undetectable 210 or prominent FRB-mCh recruitment at ER-PM junctions pre- and $1 \mathrm{~h}$ post-rapamycin addition

211 (Supplementary Fig. 8a,b, respectively). Imaging showed much slower cytosolic protein 212 recruitment compared to sFRB trimerization (12 mins vs. 1 h, Supplementary Video 1 vs.

213 Supplementary Video 2). Pairwise image correlation of the three wavelengths showed 214 significantly increased correlation coefficient $(p<0.0001)$ between the ER and PM channels, 215 increased correlation between cytosolic FRB-mCh and ER ( $p=0.030)$, and increased but not 216 significant cytosolic FRB-mCh and PM correlation $(p=0.16)$, reflecting less robust $F$ RB-mCh

217 recruitment (Supplementary Fig. 8c). Because sFKBP was less efficient compared to sFRB in 218 targeting cytosolic proteins to ER-PM junctions, we decided to continue with only sFRB for MCS 219 applications for the rest of the study.

220 We applied sFRB CIT further to recruit cytosolic proteins to ER-mitochondria junctions. 221 Complimentary sFRB pairs were expressed on the ER (sFRB $1 \mathrm{~N}-\mathrm{CFP}-\mathrm{Cb} 5$ ) and mitochondria 222 (Tom20-YFP-sFRB 1 ), and post-rapamycin addition translocated mCh-FKBP to ER-mitochondria 
223 MCS (Fig. 4c and Supplementary Video 3). Pairwise image correlation coefficients increased for

224 all three wavelengths, indicating successful trimerization ( $p<0.0001$ all cases; Fig. 4e). Likewise,

225 removal of $s F R B_{1 N}(L y n-Y F P)$ and $s F R B_{1 C}(m C h-C b 5)$ yielded no translocation of mCh-FKBP to ER-

226 mitochondria MCS after rapamycin addition (Supplementary Fig. 9a,b, left and center), whereas

227 removal of FKBP resulted in increased co-localization between the ER and mitochondria

228 components, without any co-localization of mCh (Supplementary Fig. 9a,b, right).

229 CIT induces tri-organellar membrane contact sites

230 Multi-spectral live-cell imaging of organelles has revealed that ER-mitochondria MCS make

231 frequent contact with the Golgi, peroxisomes, and lipid droplets, hinting at tri-organellar $\mathrm{MCS}^{22}$.

232 ER-mitochondria MCS also mark regions of autophagosome formation ${ }^{32}$. We therefore

233 speculated that a tri-organelle ER-PM-mitochondria junction site could exist due to the existence

234 of ER-PM, ER-mitochondria, and PM-mitochondria MCS, and their shared function in $\mathrm{Ca}^{2+}$

235 signaling. Using CIT to determine if a tri-organellar junction could be synthetically formed

236 between the PM, ER and mitochondria, we co-expressed SFRB 1 lc localized to the PM (Lyn-CFP-

$\left.237 s F R B_{1 C}\right), s F R B_{1 N}$ to the ER (YFP-sFRB $\left.1 N-C b 5\right)$ and FKBP to the mitochondria (Tom20-mCh-FKBP) in

238 Cos-7 cells (Fig. 5a-c). Fifteen minutes post-rapamycin addition, PM, ER and mitochondrial signals

239 colocalized (Fig. 5b,c and Supplementary Video 4); correlation coefficients increased between

240 PM-ER, ER-mitochondria and PM-mitochondria signals ( $p<0.0001$ all cases; Fig. 5d). When

$241 \mathrm{sFRB}_{1 \mathrm{C}}, \mathrm{sFRB} \mathrm{N}_{1 \mathrm{~N}}$, or FKBP were each removed, trimerization no longer occurred post-rapamycin

242 (Supplementary Fig. 10a,b, left and right), but removal of FKBP still resulted in colocalization of

243 ER-PM signals (Supplementary Fig. 10a,b, center). These results show that CIT can induce PM-

244 ER-mitochondria tethering, and serves as a detection method for potential tri-organellar MCS

245 formation between these three organelles.

\section{Localized $\mathrm{PI}(4,5) \mathrm{P}_{2}$ depletion at ER-PM junctions}

247 The major functions of ER-PM MCS are $\mathrm{Ca}^{2+}$ re-entry and lipid homeostasis. As $\mathrm{Ca}^{2+}$ re-entry is

248 more established, the focus in the ER-PM field has shifted in recent years towards lipid transfer

249 and homeostasis ${ }^{33}$. One major topic in the field is the regulation of secondary lipid messenger

$250 \mathrm{PI}(4,5) \mathrm{P}_{2}\left(\mathrm{PIP}_{2}\right)$ and its precursor $\mathrm{PI}(4) \mathrm{P}$. Because the $\mathrm{PM}$ is a large signaling hub of the cell 
251 converting messages from extracellular milieu to intracellular signals, PIP 2 and its precursor

$252 \mathrm{PI}(4) \mathrm{P}$ levels are often in flux. ER-PM junctions serve as a bridge connecting the PM and ER, where

253 local proteins regulate PM lipid homeostasis by using the ER as a reservoir or a sink, depending

254 on the needs of the $\mathrm{PM}^{20,31,34}$. While methods exist to perturb PM lipid composition or locally

255 deplete $\mathrm{PIP}_{2}$ from the $\mathrm{PM}^{35}$, the means of perturbing lipids only at ER-PM junctions have yet to

256 be established.

257 To achieve local $\mathrm{PIP}_{2}$ depletion, we targeted cytosolic PIP 2 phosphatase, INP54P, to ER-PM

258 junctions. PM-localized sFRB $1 \mathrm{~N}$ (Lyn-Clover-FRB $1 \mathrm{~N}$ ), ER-localized sFRB $1 \mathrm{C}$ (iRFP-FRB $1 \mathrm{C}-\mathrm{Cb}_{5}$ ),

259 cytosolic CFP-FKBP fused to 5-phosphatase INP54P (331) (CF-INP54P), and mRuby-PH-PLC $\delta$, a

260 biosensor for $\mathrm{PIP}_{2}$ were co-expressed in Cos-7 cells (Fig. 6a). After rapamycin addition, CF-INP54P

261 was recruited to ER-PM junctions, and a marked pattern of reduction in mRuby-PH-PLC $\delta$ intensity

262 was observed (Fig.6b, left, and Supplementary Video 5). In contrast, recruitment of the INP54P

263 phosphatase-dead mutant CF-INP54P D281A, or CF alone did not reduce mRuby-PH-PLC $\delta$

264 intensity (Fig.6b, center and left, and Supplementary Videos 6 and 7, respectively). Localized

$265 \mathrm{PIP}_{2}$ reduction occurred in areas of high (arrow) compared to low ER-PM junction formation

266 (chevron), reflected in percent change in mRuby-PH-PLC $\delta$ intensity in the respective regions $(p<$

267 0.0001, Fig. 6c). CF-INP54P D281A recruitment inside ER-PM rich regions was slightly decreased

268 compared to outside ( $p=0.0358$, Fig. $6 c$ ). CF recruitment inside ER-PM rich regions was not

269 significantly different compared to outside ( $p=0.869$, Fig. $6 c)$. These results show that CIT-based

270 recruitment of INP54P can locally deplete PIP 2 at ER-PM junctions.

271 


\section{Discussion}

273 In this study we designed, developed and implemented the first known CIT system by rationally 274 splitting proteins of the FKBP/FRB CID system. The CIT tool is small, robust, and trimerizes on a 275 timescale of seconds-to-minutes. We used CIT to rapidly target cytosolic protein exclusively to 276 ER-PM and ER-mitochondria MCSs, induce tri-organellar ER-mitochondria-PM junctions and 277 locally deplete PIP $_{2}$ at ER-PM MCS. We believe that our ability to achieve CIT is attributed to the 278 unique cooperativity of the FKBP/FRB CID. This efficient CIT system would be otherwise highly 279 difficult to achieve de novo, as engineering sequential cooperativity at protein interfaces would 280 be necessary.

281 The sFKBP CIT system, being slower than the sFRB CIT system, provides evidence for the 282 mechanism of trimerization. As FKBP binding affinity for rapamycin is over 10,000-fold higher 283 than FRB, FKBP-rapamycin complexes form much faster. We believe that in sFRB systems, FKBP284 rapamycin complexes are first quickly formed, then bind and stabilize sFRB halves, resulting in 285 trimerization. In contrast, in sFKBP systems, FRB-rapamycin complexes take longer to form, 286 causing slow stabilization of the trimer. It is also possible for split proteins to bind free rapamycin 287 first, and then recruit its CID partner; indeed, SFKBP can dimerize in vitro in the absence of FRB, 288 as evidenced by crystal structure analysis (Fig. 3). However, it is relatively unlikely to expect split proteins to bind rapamycin with higher affinity than its full length counterpart. Therefore our

290 results suggest that the predominant mechanism of trimerization is split protein recombination 291 mediated by the rapamycin-CID partner complex.

292 Components of sFRB and sFKBP CIT systems are small; sFRB ${ }_{1 \mathrm{~N}}$ and sFRB $1 \mathrm{c}$ are $5 \mathrm{kDa}$ and $6.3 \mathrm{kDa}$ 293 respectively, thereby increasing functionality without adding bulk. While $s F R B_{\chi N}$ and $s F R B \chi c$ 294 halves do not function orthogonally, we discovered three additional sFRB pairs (sFRB $1{ }_{1 N} / \mathrm{sFRB}_{3 \mathrm{C}}$, $295 \mathrm{sFRB}_{2 \mathrm{~N}} / \mathrm{sFRB}_{1 \mathrm{C}}, \mathrm{sFRB}_{2 \mathrm{~N}} / \mathrm{sFRB}_{3 \mathrm{C}}$ ) that trimerize with FKBP (Supplementary Fig. 6d). Thus in total, 296 we have developed 6 sFRB pairs capable of trimerization, increasing the likelihood of successful 297 CIT implementation across biological systems. Having 6 CIT systems introduces variety for future mutagenesis studies to achieve orthogonality, irreversibility and improved expression. 
299 Depletion of PIP 2 at ER-PM junctions (Fig. 6) serves as a springboard for further experiments 300 investigating differences in local $\mathrm{PIP}_{2}$ depletion at the ER-PM junction compared to global PIP 2 301 depletion at the PM; it may provide insight into the relationship between lipid transfer and $\mathrm{Ca}^{2+}$ 302 entry processes at the ER-PM junction, as they share common players such as PIP 2 and PI(4)P. In 303 a broader context, CIT-based targeting of panels of molecular actuators or proteins of interest to 304 junctions can serve as a top-down screening method to investigate local signaling. Additionally, 305 the tri-organellar PM-ER-mitochondria junction formed through CIT indirectly suggests that these 306 three organelles have the potential of interacting. In the future, CIT can be a powerful tool to 307 validate and perturb other tri-organellar MCS, such as the ER-mitochondria-Golgi junction 308 suggested by a recent study ${ }^{22}$, which is feasible to achieve due to an inherently modular nature 309 of CIT molecular design.

310 In all, we developed a trimerization tool capable of cell signaling manipulation in new ways. MCS 311 targeting in this study demonstrated the enhanced spatiotemporal specificity CIT has over CID.

312 We see potential for the tool beyond its role in future screens for studying MCS. Trimerization is 313 a common mechanism in nature, and CIT-based mimicry may deduce the significance of 314 trimerization in these systems. CIT may also produce increasingly sophisticated, tunable switches 315 and logic gates. By introducing a third component to be recruited to two others with one chemical 316 stimulus, CIT introduces a new tool to cell, chemical and synthetic biology toolkits. 


\section{Online Methods}

\section{Cell culture and transfection}

HeLa and Cos- 7 cells were maintained at $37^{\circ}$ C, $5 \%$ CO2 and 95\% humidity in Dulbecco's modified

321 Eagle's medium (DMEM) supplemented with 10\% FBS and 1\% Pen/strep (Gibco). The day prior

322 to imaging, cells were detached with 5\% Trypsin-EDTA (Gibco) and harvested for reverse 323 transfection with FuGENE HD (Promega). As a general rule, co-transfection of split and unsplit 324 proteins used a split protein to unsplit protein plasmid ratio of 20:1 for SFRB CIT experiments and 325 a ratio of 10:1 for sFKBP CIT. Prior to imaging, cell medium in samples was replaced with DPBS 326 containing $\mathrm{Ca}^{2+}$ and $\mathrm{Mg}^{2+}$ for imaging $<30$ mins or fresh DMEM growth media for imaging $>30$ 327 mins. Cells were transfected with constructs at adjusted relative plasmid ratios and incubated for 328 16-24h prior to imaging.

\section{DNA plasmid construction}

330 Constructs used to determine working split proteins were based off Clontech C1 and N1 331 backbones with various fluorophores. A 5xSAGG flexible linker oligo was annealed was inserted 332 between Kpnl and Sall restriction sites of both $\mathrm{C} 1$ and N1 backbones. Lyn or CAAX oligo DNA 333 fragments were subsequently annealed and ligated into C1 or N1 backbones containing 5xSAGG 334 linkers. Then, FRB or FKBP fragments were cloned and inserted between Kpnl and BamHI or 335 HindIII and Sall for C1 and N1 vectors respectively. To obtain non-Lyn or CAAX versions of the 336 proteins, the portion containing fluorescent protein with split FRB or FKBP fragments were cut 337 and pasted into $\mathrm{C} 1$ or $\mathrm{N} 1$ backbone vectors lacking Lyn or CAAX. YFP-sFRB $1 \mathrm{~N}-\mathrm{Cb} 5$ was generated 338 by deletion mutagenesis of previously reported YFP-FRB-Cb5 ${ }^{4}$. ER-targeting sequence Cb5 was 339 amplified by $P C R$ and subcloned into a $s F R B_{1 N}-C$ backbone from $s F R B_{1 N}-C-C b 5$ to generate 340 sFRB $_{1 \mathrm{~N}}-\mathrm{C}-\mathrm{Cb} 5$. CF-INP54P (331) and CF-INP54P (D281A) were from a previous report ${ }^{36}$. All 341 plasmids were verified by Sanger sequencing.

\section{$342 \quad$ Live-cell imaging}

343 Most imaging was conducted on a IX71 microscope (Olympus) with a 63x objective (and 1.6x 344 zoom) and an ORCA-Flash4.0 LT Digital CMOS camera (Hamamatsu). Rate constant of FKBP 
recruitment by $\mathrm{SFRB}_{1}$ was imaged at 10 s intervals with a spinning disc confocal, inverted Axiovert 200 (Zeiss) with a 40x objective and an Orca ER CCD camera (Hamamatsu). Both microscopes

347 were driven by Metamorph 7.5 imaging software (Molecular Devices). Four-color imaging in Fig.

3484 was conducted with an Eclipse Ti microscope (Nikon) with a 60x objective (and 1.5x zoom) and

349 a Zyla 4.2 sCMOS camera (Andor), driven by NIS Elements software (Nikon). Unless otherwise

350 indicated imaging was done at 1-3 min intervals for 12-30 mins, at times with between 3-5 0.5

351 um-spaced z positions. Images analyzed and shown are from a single plane, and not maximum

352 intensity projections. Microscopy experiments applying CIT to membrane contact sites were

353 conducted at $37^{\circ} \mathrm{C}, 5 \% \mathrm{CO}_{2}$ and humidity with a stage top incubation system (Tokai Hit).

\section{Analysis of PM-to-cytosol ratio}

355 PM-to-cytosol ratio was determined through linescan analysis. Lines through cells were drawn to 356 1) avoid the nucleus and 2) maximize the two membrane signals on both sides of the cell. Two 357 PM (p1, p2) locations based on peak intensities (I) along the linescan were determined visually. 358 The cytosol was defined as midpoint location $(m)$ between $p_{1}$ and $p_{2}$. A moving average of 3 was 359 used to determine signal intensity at $p_{1}$ and $p_{2}$, and a moving average of 5 at the cytosol location. 360 Then PM-to-cytosol ratio was determined for each PM location, and then averaged. Percent 361 change was determined from PM to cytosol ratio values before and after rapamycin treatment.

$$
\frac{P M}{\text { cytosol }}=\frac{5\left(\sum_{p_{1}-1}^{p_{1}+1} I+\sum_{p_{2}-1}^{p_{2}+1} I\right)}{6 \sum_{m-2}^{m+2} I}
$$

\section{Analysis of recruitment rate}

365 Averaged intensities from 3 regions of interest (ROI's) from the cytosol were used to generate 366 exponential decay curves. Averaged intensity timepoints with exponential decay trends were 367 selected for line fitting with an exponential regression to determine the exponential recruitment 368 coefficient. $R^{2}$ values were all $>0.88$. 
370 Analysis of trimerization with Fisher's transform of Pearson's correlation coefficient

3713 ROI's about $50 \times 50$ pixels were manually positioned for each cell to determine Pearson's

372 correlation using FIJI. ROls were chosen include enough background and organelle to mitigate

373 false correlations. Due to organelle movement and deformation, different ROI's were selected

374 for the same cell before and after rapamycin addition. Pairwise Pearson's correlation (between

375 ROI1 and ROI2) was determined among three channels using the built-in MATLAB function corr2,

376 and averaged between 3 ROl's. Fisher's transform was performed to normalize Pearson's

377 correlation coefficient for later statistical analysis. The result is altogether referred to as

378 correlation coefficient.

Correlation coefficient $=\tanh ^{-1}(\operatorname{corr} 2(R O I 1, R O I 2))$

\section{Statistical analysis and data visualization}

381 Unless otherwise stated, most statistical analysis was done with unpaired, two-tailed Student's

382 T-test assuming equal distributions. A paired, two-tailed Student's T-test was used in Fig. 6c. 383 PRISM 6 software was used for statistical analysis and data visualization.

\section{Split site prediction}

385 Split sites were determined by selecting non-evolutionarily conserved solvent accessible loops.

386 The Stride program ${ }^{37}$ was used to calculate the solvent accessible areas (SAA). Loops exceeding $38730 \AA^{2}$ were selected as potential split sites. Sequence conservation as indicated by Kullback-

388 Liebler conservation scores was determined with MISTIC ${ }^{38}$ and FKBP domain and FRB domain 389 sequences used were obtained from PFAM server ${ }^{39}$ (Finn 2014). Loops with Kullback-Leibler

390 conservation scores less than 2 were selected as potential split sites. The difference between the 391 native energy of the intact protein and the sum of the energies of the split proteins at a given 392 split site - the split energy - was calculated using the MEDUSA scoring function ${ }^{40}$ (Yin 2007). 393 Energy wells were indicative of split sites in this study, which differs from the previous 394 implementation of SPELL, which targeted regions outside energy wells ${ }^{41}$. The original SPELL study 395 looked for split proteins sites which minimized the destabilization as these proteins were then 
396 fused to a known destabilizer domain (iFKBP) ${ }^{41}$. This approach ensures the prevention of the split 397 part association in the absence of rapamycin.

\section{Crystallography}

\section{Protein expression and purification}

401 For structural analysis, cDNA encoding the sFKBP $_{1 N}$ (residues 1-32 of human FKBP12) was 402 amplified by PCR and subcloned into a pET28a(+) derivative vector, which has a maltose binding 403 protein (MBP)-encoding sequence inserted after an $\mathrm{N}$-terminal polyhistidine tag. The construct 404 contained a tobacco etch virus (TEV) protease recognition site at the junction between 405 polyhistidine tag and the MBP sequences. Recombinant sFKBP $1 \mathrm{~N}$ protein was expressed in E. coli $406 \mathrm{BL21}(\mathrm{DE} 3)$ cells in $\mathrm{LB}$ broth at $37{ }^{\circ} \mathrm{C}$ until the $\mathrm{OD}_{600}$ reached 0.8 , at which point the temperature 407 was shifted to $18{ }^{\circ} \mathrm{C}$. Protein expression was induced by addition of isopropyl- $\beta$-D408 thiogalactopyranoside to a final concentration of $250 \mu \mathrm{M}$. The cultures were grown for an 409 additional $20 \mathrm{hrs}$ and harvested by centrifugation. Cell pellets were resuspended in $50 \mathrm{mM}$ Tris$410 \mathrm{HCl}$ buffer ( $\mathrm{pH}$ 7.0) containing $500 \mathrm{mM} \mathrm{NaCl}, 20 \mathrm{mM}$ imidazole and 10\% glycerol. Cells were lysed 411 by sonication and clarified by centrifugation. The cell lysate was loaded onto a HisTrap HP column 412 (GE Healthcare), and eluted with $50 \mathrm{mM}$ Tris-HCl buffer (pH 7.0) containing $500 \mathrm{mM} \mathrm{NaCl}, 500$ $413 \mathrm{mM}$ imidazole and $10 \%$ glycerol. The $\mathrm{N}$-terminal histidine tag was cleaved by incubating with TEV 414 protease overnight at $4{ }^{\circ} \mathrm{C}$. The protein was further purified using a HiLoad Superdex 200 16/60 415 size-exclusion column (GE Healthcare) equilibrated with $20 \mathrm{mM}$ Tris- $\mathrm{HCl}$ buffer (pH 8.0) 416 containing $150 \mathrm{mM} \mathrm{NaCl}$ and $5 \mathrm{mM}$ DTT. The sFKBP $1 \mathrm{c}$ (residues 33-108 of human FKBP12) was 417 expressed and purified essentially as the same as SFKBP $1 \mathrm{~N}$, except an N-terminal His-SUMO tag 418 was fused instead of the polyhistidine and MBP. The His-SUMO tag was cleaved by SUMO 419 protease overnight at $4{ }^{\circ} \mathrm{C}$. The purified $s F K B P_{1 N}$ and $s F K B P_{1 c}$ proteins were mixed in a 1:2 molar 420 ratio, and then the mixture was added to a $1 \mu \mathrm{M}$ rapamycin solution in $20 \mathrm{mM}$ Tris- $\mathrm{HCl}$ buffer $421\left(\mathrm{pH}\right.$ 8.0) containing $150 \mathrm{mM} \mathrm{NaCl}, 5 \mathrm{mM}$ DTT and 10\% DMSO. The sFKBP $1 \mathrm{~N}, \mathrm{sFKBP}_{1 \mathrm{c}}$ and 422 rapamycin mixture was filtered and loaded onto a Superdex 200 Increase 10/300 column (GE 423 Healthcare) equilibrated with $20 \mathrm{mM}$ Tris- $\mathrm{HCl}$ buffer $(\mathrm{pH} 8.0)$ containing $150 \mathrm{mM} \mathrm{NaCl}$ and $5 \mathrm{mM}$ 
424 DTT. The eluted peak fractions of $\mathrm{sFKBP}_{1} \cdot$ rapamycin complex were pooled and concentrated to $42540 \mathrm{mg} / \mathrm{ml}$. The cDNAs encoding human FKBP12 (residues 1-108) and FKBP12-rapamycin binding 426 (FRB) domain encompassing residues 2021-2113 of human mTOR (T2098L) were subcloned into $427 \mathrm{pET} 28 \mathrm{a}(+)$ containing an N-terminal polyhistidine-GST tag. The FKBP12 and FRB mutant proteins 428 were expressed and purified essentially as the same as SFKBP $1 \mathrm{~N}$. The His-GST tag was cleaved by 429 TEV protease overnight at $4{ }^{\circ} \mathrm{C}$.

431 Crystallization was performed by the sitting-drop vapor-diffusion method at $20{ }^{\circ} \mathrm{C}$. Crystals of $432 \mathrm{SFKBP}_{1}$.rapamycin appeared by mixing equal volumes of a $40 \mathrm{mg} / \mathrm{ml} \mathrm{sFKBP}{ }_{1}$.rapamycin complex 433 solution and a reservoir solution of $100 \mathrm{mM}$ Na-HEPES buffer $(\mathrm{pH} 7.5)$ containing 20\% (w/v) PEG 434 8000. FKBP12-rapamycin·FRB (T2098L) complex was prepared by mixing them at an equimolar 435 ratio and incubating for 60 min on ice. Crystals of FKBP12-rapamycin·FRB (T2098L) appeared at $436100 \mathrm{mM}$ cacodylic acid buffer (pH 6.5) containing $350 \mathrm{mM}$ zinc acetate and 8\% (w/v) isopropanol. 437 sFKBP 1 rapamycin·FRB (T2098L) complex was prepared in the same manner as 438 FKBP12-rapamycin·FRB (T2098L). Crystals of sFKBP ${ }_{1} \cdot$ rapamycin·FRB (T2098L) appeared at 100 $439 \mathrm{mM}$ Tris- $\mathrm{HCl}$ buffer $(\mathrm{pH}$ 7.0) containing $200 \mathrm{mM}$ calcium acetate and $20 \%(\mathrm{w} / \mathrm{v})$ PEG 3000. All 440 crystals were briefly soaked in a cryoprotectant drop composed of the reservoir solution 441 supplemented with $20 \%$ glycerol and then flash-cooled in liquid nitrogen for X-ray diffraction 442 data collection. The datasets were collected at $1.000 \AA$, $100 \mathrm{~K}$ on the beamline BL26B2 at SPring4438 (Harima, Japan) or the beamline X06DA at the Swiss Light Source (Villigen, Switzerland). They 444 were processed using the XDS ${ }^{42}$ and scaled using AIMLESS supported by other programs of the 445 CCP4 suite ${ }^{43}$. Crystal structures were determined by molecular replacement using MOLREP 44 446 with the FKBP12-rapamycin·FRB structure (PDB ID: 1FAP) and the MBP structure (PDB ID: 1ANF) 447 as the search models. Model building was accomplished with $\operatorname{Coot}^{45}$, and structural refinement 448 was performed with REFMAC ${ }^{46}$ and PHENIX ${ }^{47}$. The Ramachandran statistics are as follows: $96.0 \%$ 449 favored, 4.0\% allowed for $\mathrm{SFKBP}_{1} \cdot$ rapamycin; $97.2 \%$ favored, $2.8 \%$ allowed for 450 FKBP12·rapamycin·FRB (T2098L); and 96.8\% favored, 3.2\% allowed for sFKBP 1 -rapamycin·FRB 451 (T2098L). The data collection and refinement statistics are summarized in Supplementary Table 
bioRxiv preprint doi: https://doi.org/10.1101/2020 03.16.994277; this version posted March 18, 2020. The copyright holder for this preprint (which was not certified by peer review) is the author/funder, who has granted bioRxiv a license to display the preprint in perpetuity. It is made available under aCC-BY-NC-ND 4.0 International license.

452 1. The structural models in the figures were depicted using PyMOL version 1.8 software 453 (Schrödinger, LLC). Two-dimensional interaction plots were carried out with LIGPLOT ${ }^{48}$. 


\section{Acknowledgements}

456 We thank Luca Bertozzi and Sarah Thompson for help with plasmid generation. We thank Robert

457 DeRose, Xinyi Y. Zhou, and Yuta Nihongaki for proofreading the manuscript. We thank Hideaki

458 Niwa (RIKEN), Naoki Sakai (RIKEN), and the staff at the BL26B2 beamline (Proposal No. 20190047)

459 of SPring-8 (Harima, Japan) and the X06DA beamline (Proposal No. 20171001) of the Swiss Light

460 Source, Paul Scherrer Institut (Villigen, Switzerland) for their help in X-ray diffraction data

461 collection. We acknowledge support from the National Institutes for Health (5R01GM123130 to

462 T.I., and 5R01GM123247 and 1R35 GM134864 to N.V.D.), the Passan Foundation to N.V.D., the 463 DoD DARPA (HR0011-16-C-0139 to T.I.), and the PRESTO program of the Japan Science and 464 Technology Agency to T.U. (No. JPMJPR12A3) and T.I (No. JPMJPR12A5), and a Grant-in-Aid for 465 Scientific Research (B) to T.U. (No. 16H05089) from the Japan Society for the Promotion of 466 Science.

\section{Author contributions}

468 H.D.W. and H.N. conceived the study with input from T.I. H.D.W. carried out cell experiments and 469 conducted image analysis with help from A.K.A. M.K. purified and crystalized split proteins, and 470 determined protein structure by X-ray crystallography. O.D. conducted rational split site analysis. 471 T.I., H.N., T.U., and N.V.D. supervised the project. H.D.W. wrote the manuscript in consultation 472 with T.I. and with input from M.K., T.U., O.D., and N.V.D.

\section{Competing Interests}

474 There is an ongoing disclosure associated with the CIT tools.

475

476 


\section{$477 \quad$ Figure Legends}

478 Figure 1: Rational CIT design and sFRB overexpression in cells. (a) CID proteins FRB (green, arc)

479 sites generates FRB protein halves (yellow and blue). Split FRB halves and FKBP form three

480 components of CIT. Rapamycin brings them together. (b) Computationally analysis to determine

481 split sites for FRB domain only. Loop areas with high solvent accessibility area (SAA), low

482 conservation and favorable split energy profiles were chosen. (c) FRB structure (PDB ID: 1AUE)

483 consists of $4 \alpha$-helices; split sites 1, 2 and 3 all reside in linker regions between $\alpha$-helices. (d)

484 Examples of sFRB fusion protein design. Lyn or CAAX motifs target sFRBs to the PM. (e) Co-

485 overexpression of sFRB protein pairs in HeLa cells. N-terminal sFRB halves (sFRB $1 \mathrm{~N}, s F R B_{2 N}$,

$486 \mathrm{sFRB}_{3 \mathrm{~N}}$ ) are PM localized by Lyn and C-terminal sFRB halves (sFRB $\left.1 \mathrm{c}, \mathrm{sFRB}_{2 \mathrm{C}}, \mathrm{sFRB} 3 \mathrm{C}\right)$ are

487 cytosolic. Linescans below show normalized PM (orange) and cytosolic (blue) signal intensity. (f)

488 Pre-association between sFRB halves. PM-to-cytosolic ratios derived from linescans of cytosolic

$489 \mathrm{sFRB}_{1 \mathrm{C}}, \mathrm{sFRB}_{2 \mathrm{C}}$, and $\mathrm{sFRB} 3 \mathrm{C}$ co-expressed with PM-targeted $\mathrm{sFRB} \mathrm{B}_{1 \mathrm{~N}}, \mathrm{sFRB}_{2 \mathrm{~N}}$, and $\mathrm{sFRB} \mathrm{B}_{3 \mathrm{~N}}$

490 respectively compared to CFP alone (from left to right: $n=39,33,27,39,36$, and 26 cells; 3

491 experiments). Scale bar, $10 \mu \mathrm{m}$.

493 Figure 2: Characterization of FKBP recruitment extent and kinetics by sFRB after rapamycin

494 addition. (a) Experimental setup for FKBP recruitment efficacy by sFRBs. sFRB N-terminal and C-

495 terminal halves are PM-targeted by CAAX or Lyn respectively and co-expressed with FKBP.

496 Cytosol-to-PM translocation by FKBP indicates successful recruitment. (b) Translocation of FKBP

497 from cytosol to PM in HeLa cells co-expressing PM-targeted sFRB $1, \mathrm{SFRB}_{2}, \mathrm{sFRB}_{3}$ pairs and full 
498 length FRB. Pre-rapamycin; top 3 rows. 9 min post-rapamycin; bottom row. Linescans show

499 corresponding normalized intensity of mCh-FKBP signal. (c) Percent change in mCh-FKBP PM-to-

500 cytosolic ratio pre- and post-rapamycin addition. FKBP recruitment by sFRB pairs $(\mathrm{N}+\mathrm{C})$

501 compared to $\mathrm{N}$ or C-terminal halves only ( $\mathrm{N}$ or $\mathrm{C}$ ), full length FRB or no FRB in FKBP recruitment

502 (from left to right: $n=31,29,24,34,20,24,27,23,34,47$, and 43 cells; 3-6 experiments). (d)

503 FKBP recruitment rate constants derived from exponential regression of cytosolic FKBP signal

504 decay imaged over 10 s intervals. sFRB $1 \mathrm{C}+\mathrm{sFRB} 1 \mathrm{~N}$ and FRB groups use identical plasmid

505 constructs as leftmost and rightmost columns respectively in (b) (from left to right: $n=9$ and 8;

506 4-5 experiments). Scale bar, $5 \mu \mathrm{m}$.

Figure 3: Crystal structure of the split and unsplit FKBP·rapamycin·FRB (T2098L) complexes

510 and magenta) with that of FKBP12-rapamycin·FRB (wild type; PDB ID: 1FAP, gray). T2098L is

511 shown in orange. (b) Zoom-in view of the rapamycin-interacting region of

512 FKBP12-rapamycin·FRB complexes (color-coded as in panel a). Hydrogen bonds are shown as

513 dashes. (c) Ribbon (left) and topology (right) diagrams of the sFKBP 1 (cyan), FKBP $1 \mathrm{c}$ (yellow)

514 and rapamycin (green) complex. (d) Crystal structure of sFKBP1·rapamycin·FRB (T2098L). Left,

515 sFKBP1 (cyan and yellow), rapamycin (green) and FRB (T2098L, magenta) is depicted as a ribbon

516 diagram. Right, Electron density of rapamycin. Rapamycin is depicted as a stick model with a

517 2Fo-Fc electron density (blue mesh) contoured at $1.5 \sigma$. Residues of sFKBP $1 \mathrm{c}$ (yellow) and FRB

518 (T2098L) (magenta) involved in the interaction with rapamycin are labeled and shown as a line 
519 model. (e) LIGPLOT diagrams showing critical contacts around rapamycin. Left,

520 FKBP12·rapamycin·FRB (T2098L). Right, sFKBP 1 -rapamycin·FRB (T2098L). Rapamycin (green) and

521 key residues of unsplit (left) and split (right) FKBP proteins (yellow; Y27 of sFKBP $1 \mathrm{~N}$ in cyan) and

522 those of FRB (magenta; T2098L in orange) are depicted in a ball and stick model. Carbon,

523 nitrogen and oxygen are gray, blue and red balls, respectively. Hydrogen bond and hydrophobic

524 interaction is shown in a black dashed line and a red arc of a circle, respectively. (f) Percent

525 change in FRB-mCh PM-to-cytosolic ratio pre- and post-rapamycin addition. Each sFKBP pair

$526(\mathrm{~N}+\mathrm{C})$ is compared with $\mathrm{N}$ or $\mathrm{C}$-terminal halves only $(\mathrm{N}$ or $\mathrm{C})$, full length FKBP or no FKBP in FRB

527 recruitment (from left to right: $n=21,31,38,32,39,38,28,27,21,28,33,32$, 29, and 31 cells;

$528 \quad 3-13$ experiments).

530 Figure 4: CIT-based recruitment of cytosolic protein to ER-PM and ER-mitochondria MCS. (a)

531 Targeting cytosolic FKBP (magenta) to ER-PM or ER-mitochondria MCS. sFRB halves (cyan and

532 yellow) targeted to two organelles recruit FKBP in a rapamycin-dependent manner. (b) Cos-7

533 cells expressing mCh-sFRB $1 \mathrm{C}-\mathrm{Cb} 5$, Lyn-Y-sFRB $1 \mathrm{~N}$ and CF pre- and 12 min post-rapamycin for CF

534 recruitment at ER-PM MCS. (c) Cos-7 cells expressing sFRB $1 \mathrm{~N}-\mathrm{C}-\mathrm{Cb} 5$, Tom20-Y-sFRB $1 \mathrm{c}$, and CF

535 pre- and 12 min post-rapamycin for CF recruitment at ER-mitochondria MCS. (d, e) Quantifying

536 trimerization between the 3 signals pre- and post-rapamycin. Check marks specify each

537 combination of two wavelengths used in calculating pairwise Fisher's transformation of

538 Pearson's correlation coefficients. Student's t-test was used to compare correlations pre- and

539 post- rapamycin (d, e: $n=38,27 ; 3$ experiments). Scale bars, $10 \mu \mathrm{m}$. 
541 Figure 5: Tri-organellar junction formation by CIT. (a) Trimerization of CIT components

542 targeted to ER, mitochondria and PM by rapamycin. (b, c) Cos-7 cell expressing $\mathrm{Y}-\mathrm{sFRB} \mathrm{BN}_{\mathrm{N}}-\mathrm{Cb} 5$,

543 Tom20-mCh-FKBP and Lyn-C-sFRB 1 c pre- and 15 mins post-rapamycin. (d) Quantification of

544 trimerization extent through pairwise correlation of Fisher's transformation of Pearson's

545 correlation coefficient between three wavelengths ( $n=23$ cells; 4 experiments). Scale bars, 5

$546 \mu \mathrm{m}$.

548 Figure 6: CIT induces local PI(4,5) $\mathbf{P}_{2}$ depletion at ER-PM MCS. (a) Schematic of CIT-induced

$549 \mathrm{PI}(4,5)_{2}$ depletion. CF-INP54P is recruited to ER-PM MCS by ER and PM localized sFRB halves.

550 Upon junction formation (middle panel), INP54P accesses PM PI(4,5) $\mathrm{P}_{2}$ leading to local PI(4,5) $\mathrm{P}_{2}$

551 depletion (right panel). (b) Comparing effects of ER-PM MCS recruitment of functional CF-

552 INP54P (331) against phosphatase-dead mutant CF-INP54P (D281A) and CF alone. Cos-7 cells

553 also express Lyn-Clover-sFRB $1 \mathrm{~N}$, iRFP-sFRB $1 \mathrm{c}-\mathrm{Cb}_{5}$ and mRuby $\mathrm{PH}-\mathrm{PLC} \delta$, a $\mathrm{PI}(4,5) \mathrm{P}_{2}$ biosensor.

554 Time-lapse epifluorescence microscopy taken pre- and 5 mins post rapamycin addition. (c)

555 Intensity of mRuby PH-PLCS PI(4,5) $\mathrm{P}_{2}$ biosensor taken from regions of inside (arrow) and

556 outside (chevron) ER-PM MCS, significance analyzed with paired Student's t-test (from left to

557 right: $n=28,25$ and 23 cells; each 4 experiments). Scale bar, $10 \mu \mathrm{m}$. 


\section{References}

560 1. Fegan, A., White, B., Carlson, J.C. \& Wagner, C.R. Chemically controlled protein

561 assembly: techniques and applications. Chem Rev 110, 3315-3336 (2010).

562 2. DeRose, R., Miyamoto, T. \& Inoue, T. Manipulating signaling at will: chemically-inducible

563 dimerization (CID) techniques resolve problems in cell biology. Pflugers Arch 465, 409-

$564 \quad 417(2013)$.

565 3. Spencer, D.M., Wandless, T.J., Schreiber, S.L. \& Crabtree, G.R. Controlling signal

566 transduction with synthetic ligands. Science 262, 1019-1024 (1993).

567 4. Komatsu, T. et al. Organelle-specific, rapid induction of molecular activities and

568 membrane tethering. Nat Methods 7, 206-208 (2010).

$5695 . \quad$ Haruki, H., Nishikawa, J. \& Laemmli, U.K. The anchor-away technique: rapid, conditional

570 establishment of yeast mutant phenotypes. Mol Cell 31, 925-932 (2008).

571 6. Putyrski, M. \& Schultz, C. Protein translocation as a tool: The current rapamycin story.

$572 \quad$ FEBS Lett 586, 2097-2105 (2012).

573 7. Miyamoto, T. et al. Rapid and orthogonal logic gating with a gibberellin-induced

574 dimerization system. Nat Chem Biol 8, 465-470 (2012).

575 8. Stanton, B.Z., Chory, E.J. \& Crabtree, G.R. Chemically induced proximity in biology and

$576 \quad$ medicine. Science 359 (2018).

577 9. Lambright, D.G. et al. The $2.0 \mathrm{~A}$ crystal structure of a heterotrimeric G protein. Nature $578 \quad 379,311-319$ (1996).

579 10. Higashi, T. \& Miller, A.L. Tricellular junctions: how to build junctions at the TRICkiest

580 points of epithelial cells. Mol Biol Cell 28, 2023-2034 (2017).

581 11. Hennecke, J. \& Wiley, D.C. T cell receptor-MHC interactions up close. Cell 104, 1-4 $582 \quad$ (2001).

583 12. Banaszynski, L.A., Liu, C.W. \& Wandless, T.J. Characterization of the FKBP.rapamycin.FRB $584 \quad$ ternary complex. J Am Chem Soc 127, 4715-4721 (2005).

585 13. Choi, J., Chen, J., Schreiber, S.L. \& Clardy, J. Structure of the FKBP12-rapamycin complex 586 interacting with the binding domain of human FRAP. Science 273, 239-242 (1996). 
587 14. Stankunas, K. et al. Rescue of degradation-prone mutants of the FK506-rapamycin binding (FRB) protein with chemical ligands. Chembiochem 8, 1162-1169 (2007).

15. Dagliyan, O. et al. Computational design of chemogenetic and optogenetic split proteins. Nat Commun 9, 4042 (2018).

16. Dagliyan, O. et al. Engineering extrinsic disorder to control protein activity in living cells. Science 354, 1441-1444 (2016).

17. Dagliyan, O., Dokholyan, N.V. \& Hahn, K.M. Engineering proteins for allosteric control by light or ligands. Nat Protoc 14, 1863-1883 (2019).

18. Belshaw, P.J., Schoepfer, J.G., Liu, K.Q., Morrison, K.L. \& Schreiber, S.L. Rational Design in English 34, 2129-2132 (1995).

19. Varnai, P., Toth, B., Toth, D.J., Hunyady, L. \& Balla, T. Visualization and manipulation of

20. Dickson, E.J. et al. Dynamic formation of ER-PM junctions presents a lipid phosphatase to regulate phosphoinositides. J Cell Biol 213, 33-48 (2016).

21. Phillips, M.J. \& Voeltz, G.K. Structure and function of ER membrane contact sites with other organelles. Nat Rev Mol Cell Biol 17, 69-82 (2016).

23. Prinz, W.A. Bridging the gap: membrane contact sites in signaling, metabolism, and

22. Valm, A.M. et al. Applying systems-level spectral imaging and analysis to reveal the

612 25. Liou, J. et al. STIM is a Ca2+ sensor essential for Ca2+-store-depletion-triggered Ca2+ 613 influx. Curr Biol 15, 1235-1241 (2005).

614 26. Rizzuto, R. et al. Close contacts with the endoplasmic reticulum as determinants of mitochondrial Ca2+ responses. Science 280, 1763-1766 (1998). 
616 27. Levine, T. \& Loewen, C. Inter-organelle membrane contact sites: through a glass, darkly. $617 \quad$ Curr Opin Cell Biol 18, 371-378 (2006).

618 28. Friedman, J.R. et al. ER tubules mark sites of mitochondrial division. Science 334, 358$619362(2011)$.

620 29. Rowland, A.A., Chitwood, P.J., Phillips, M.J. \& Voeltz, G.K. ER contact sites define the 621 position and timing of endosome fission. Cell 159, 1027-1041 (2014).

622 30. Helle, S.C. et al. Organization and function of membrane contact sites. Biochim Biophys $623 \quad$ Acta 1833, 2526-2541 (2013).

624 31. Zewe, J.P., Wills, R.C., Sangappa, S., Goulden, B.D. \& Hammond, G.R. SAC1 degrades its 625 lipid substrate PtdIns4P in the endoplasmic reticulum to maintain a steep chemical 626 gradient with donor membranes. Elife 7 (2018).

627 32. Hamasaki, M. et al. Autophagosomes form at ER-mitochondria contact sites. Nature $628 \quad 495,389-393(2013)$.

629 33. Saheki, Y. \& De Camilli, P. Endoplasmic Reticulum-Plasma Membrane Contact Sites. $630 \quad$ Annu Rev Biochem 86, 659-684 (2017).

631 34. Chang, C.L. \& Liou, J. Phosphatidylinositol 4,5-Bisphosphate Homeostasis Regulated by 632 Nir2 and Nir3 Proteins at Endoplasmic Reticulum-Plasma Membrane Junctions. J Biol 633 Chem 290, 14289-14301 (2015).

634 35. Idevall-Hagren, O., Dickson, E.J., Hille, B., Toomre, D.K. \& De Camilli, P. Optogenetic 635 control of phosphoinositide metabolism. Proc Natl Acad Sci U S A 109, E2316-2323 $636 \quad$ (2012).

637 36. Suh, B.C., Inoue, T., Meyer, T. \& Hille, B. Rapid chemically induced changes of 638 Ptdlns(4,5)P2 gate KCNQ ion channels. Science 314, 1454-1457 (2006).

639 37. Heinig, M. \& Frishman, D. STRIDE: a web server for secondary structure assignment from 640 known atomic coordinates of proteins. Nucleic Acids Research 32, W500-W502 (2004).

641 38. Colell, E.A., Iserte, J.A., Simonetti, F.L. \& Marino-Buslje, C. MISTIC2: comprehensive 642 server to study coevolution in protein families. Nucleic Acids Research 46, W323-W328 643 (2018). 
644 39. Finn, R.D. et al. Pfam: the protein families database. Nucleic Acids Research 42, D222-

645 D230 (2014).

646 40. Yin, S.Y., Ding, F. \& Dokholyan, N.V. Eris: an automated estimator of protein stability.

$647 \quad$ Nature Methods 4, 466-467 (2007).

648 41. Dagliyan, O. et al. Computational design of chemogenetic and optogenetic split proteins.

$649 \quad$ Nature Communications 9 (2018).

650 42. Kabsch, W. Automatic Processing of Rotation Diffraction Data from Crystals of Initially

651 Unknown Symmetry and Cell Constants. J Appl Crystallogr 26, 795-800 (1993).

652 43. Winn, M.D. et al. Overview of the CCP4 suite and current developments. Acta

$653 \quad$ Crystallogr D 67, 235-242 (2011).

654 44. Vagin, A. \& Teplyakov, A. Molecular replacement with MOLREP. Acta Crystallographica

655 Section D-Biological Crystallography 66, 22-25 (2010).

$65645 . \quad$ Emsley, P., Lohkamp, B., Scott, W.G. \& Cowtan, K. Features and development of Coot.

657 Acta Crystallographica Section D-Biological Crystallography 66, 486-501 (2010).

658 46. Murshudov, G.N., Vagin, A.A. \& Dodson, E.J. Refinement of macromolecular structures

659 by the maximum-likelihood method. Acta Crystallogr D 53, 240-255 (1997).

660 47. Adams, P.D. et al. PHENIX: a comprehensive Python-based system for macromolecular 661 structure solution. Acta Crystallogr D 66, 213-221 (2010).

662 48. Laskowski, R.A. \& Swindells, M.B. LigPlot+: multiple ligand-protein interaction diagrams 663 for drug discovery. J Chem Inf Model 51, 2778-2786 (2011). 
Figure 1

a

$$
\text { Chemically inducible dimerization (CID) }
$$

Rapa

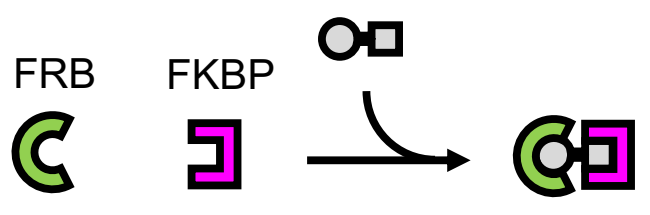

Rational protein splitting

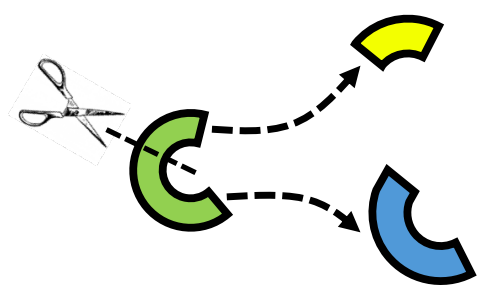

\section{Chemically inducible trimerization (CIT)}
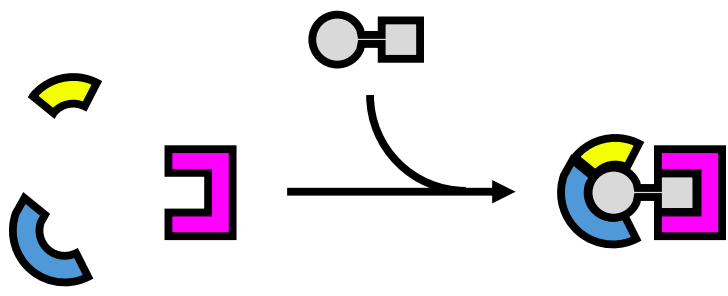

b

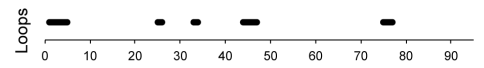

\&
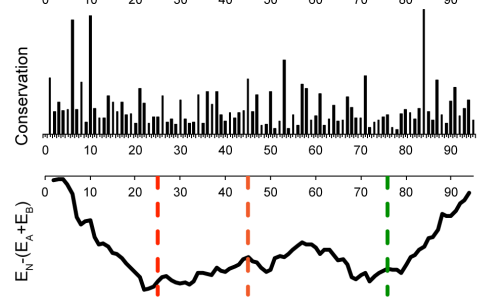

Site 3 Site 1
C

Site 3

aa. 2042-2043

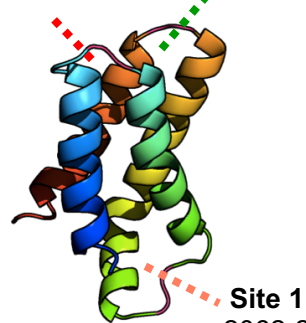

aa. $2062-2063$

Site 2 d

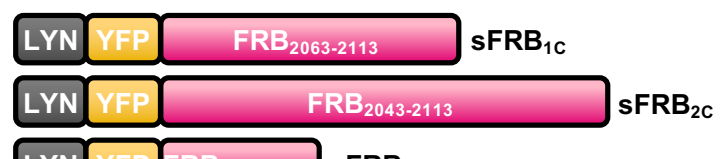

\begin{tabular}{|l|l|l|l|l}
\hline LYN & $Y F P$ & $F B_{2093-2113}$ & sFRB \\
& &
\end{tabular}

sFRB $_{1 \mathrm{~N}}$

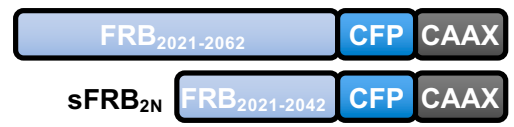

\begin{tabular}{lll} 
sFRB $_{3 N}$ & FRB \\
\hline
\end{tabular} e
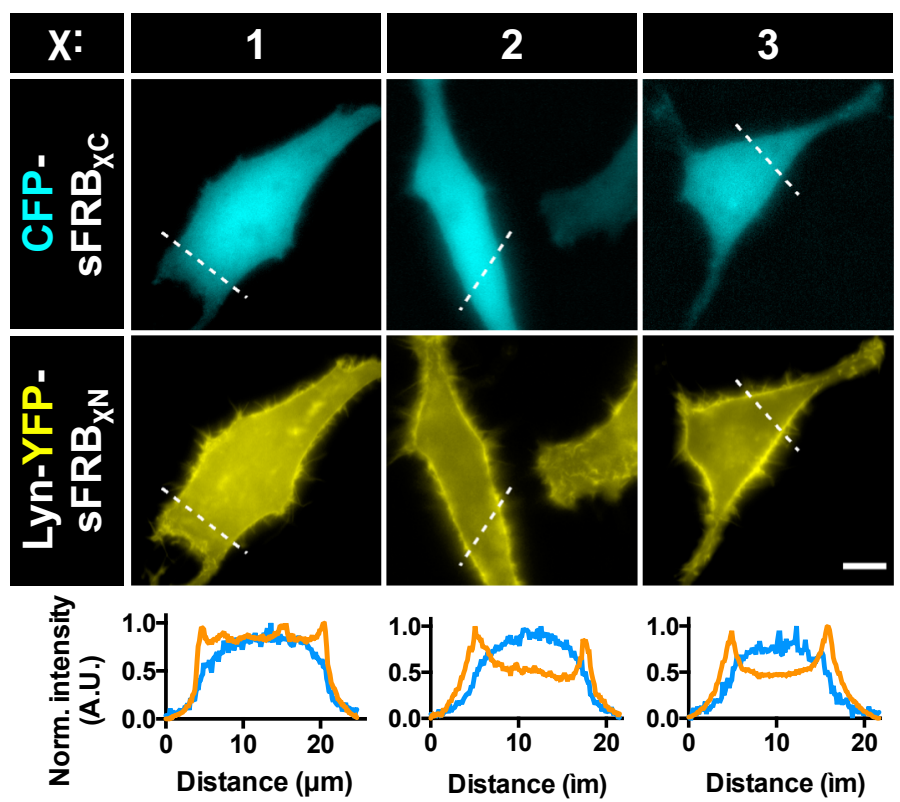

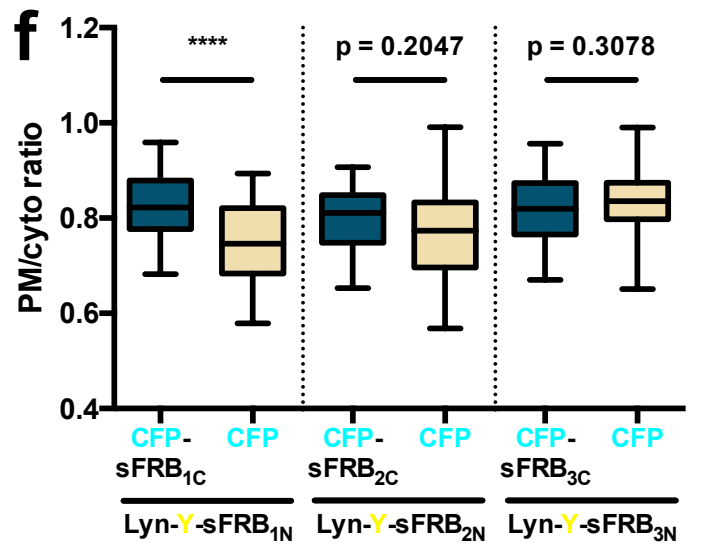


Figure 2

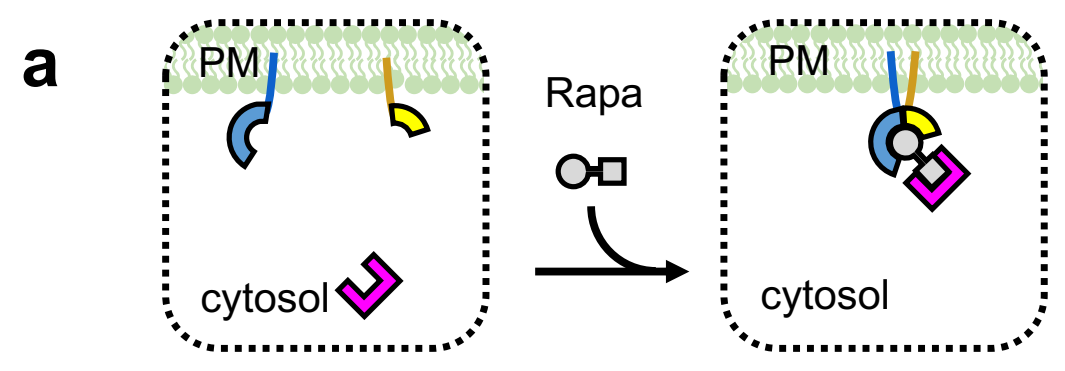

b
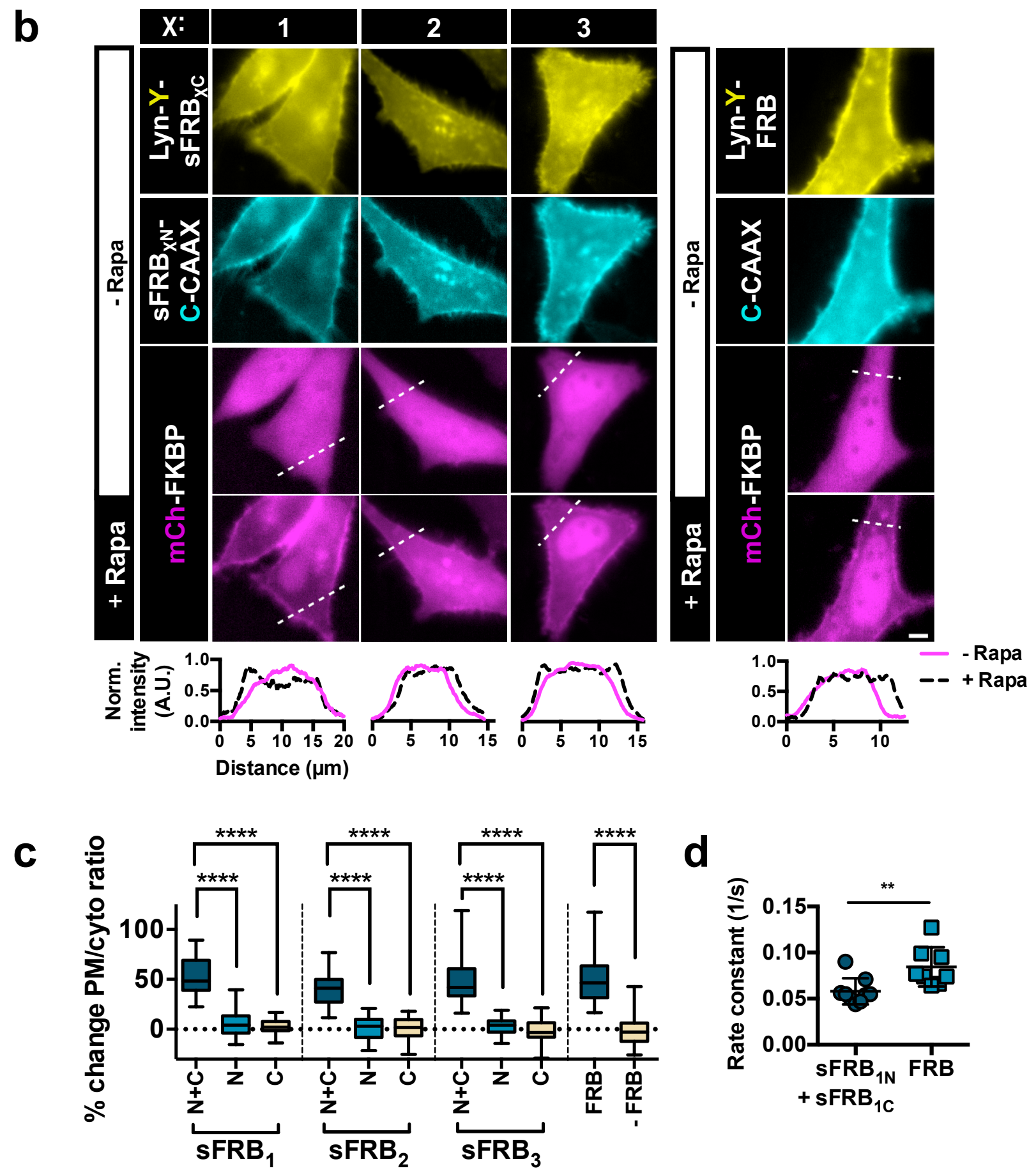

d

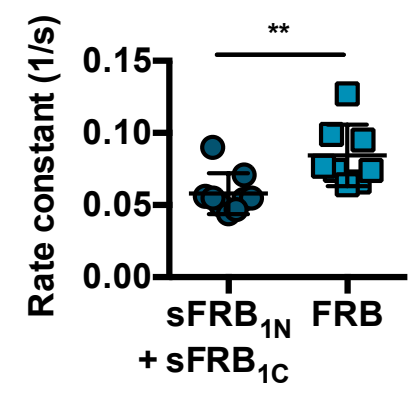




\section{Figure 3}

a

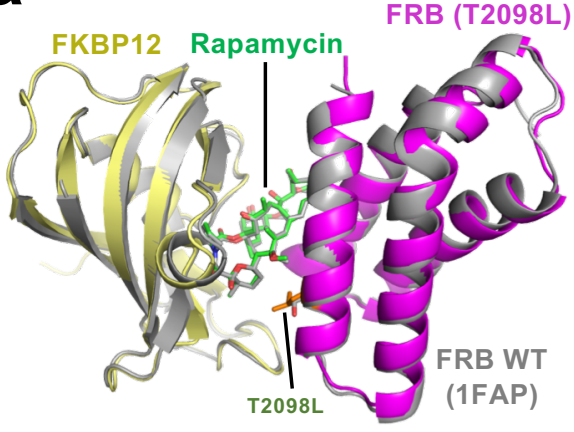

b

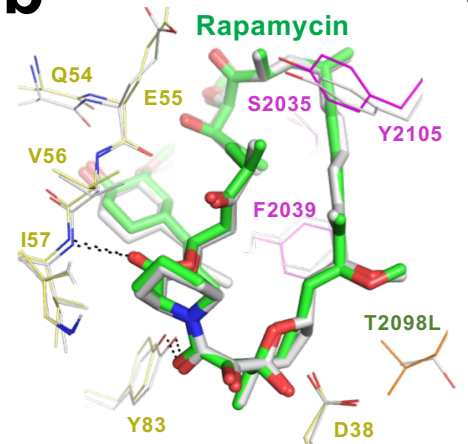

C $\mathrm{sFKBP}_{1 \mathrm{~N}} \mathrm{SFKBP} \mathrm{P}_{1 \mathrm{C}}$

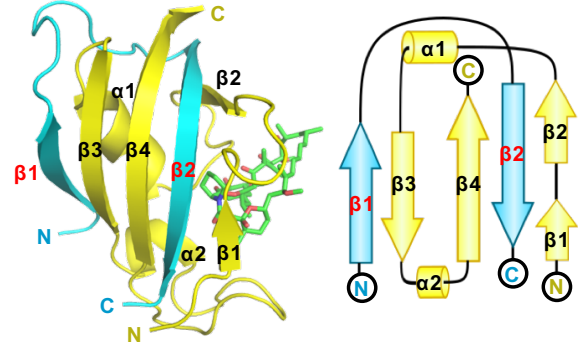

d

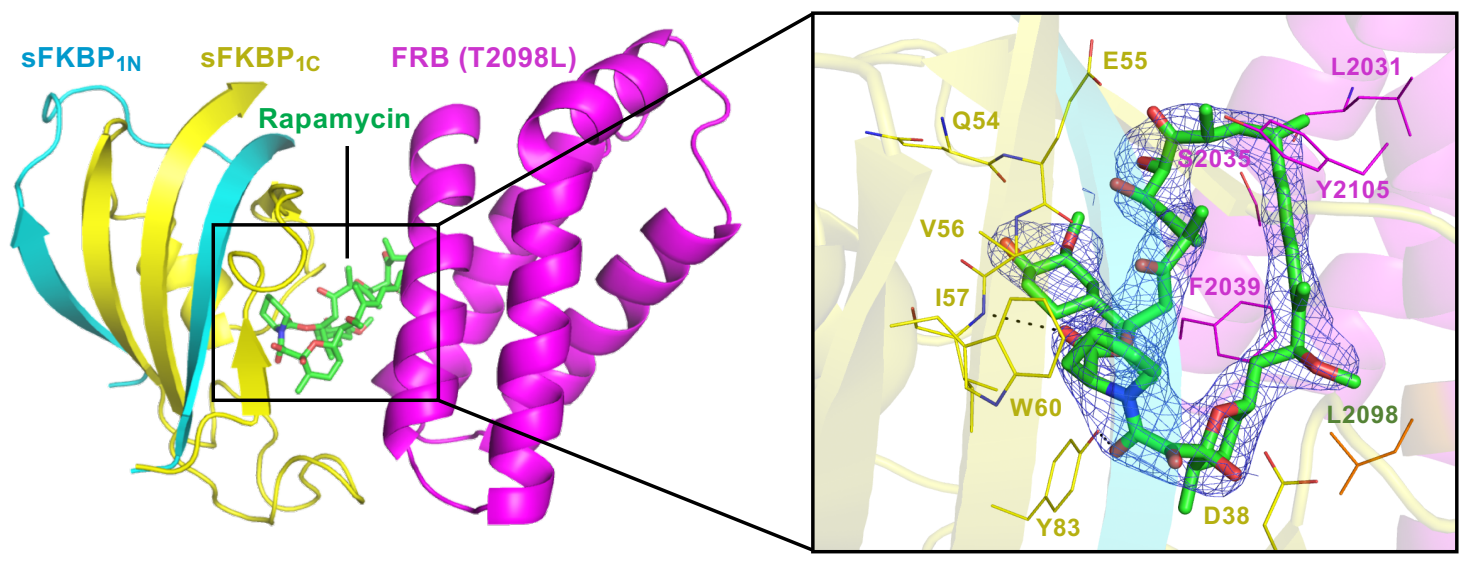

e
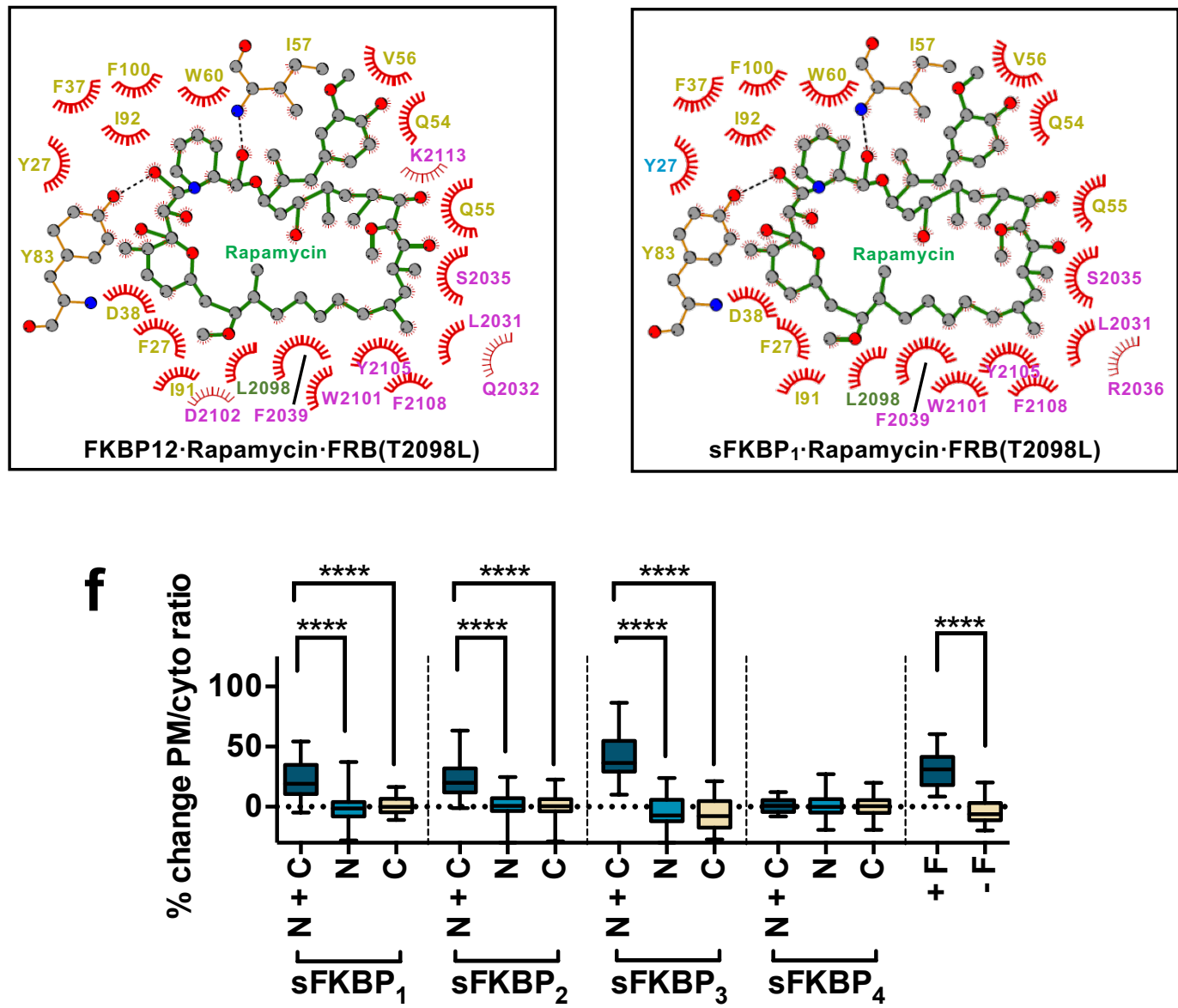
Figure 4
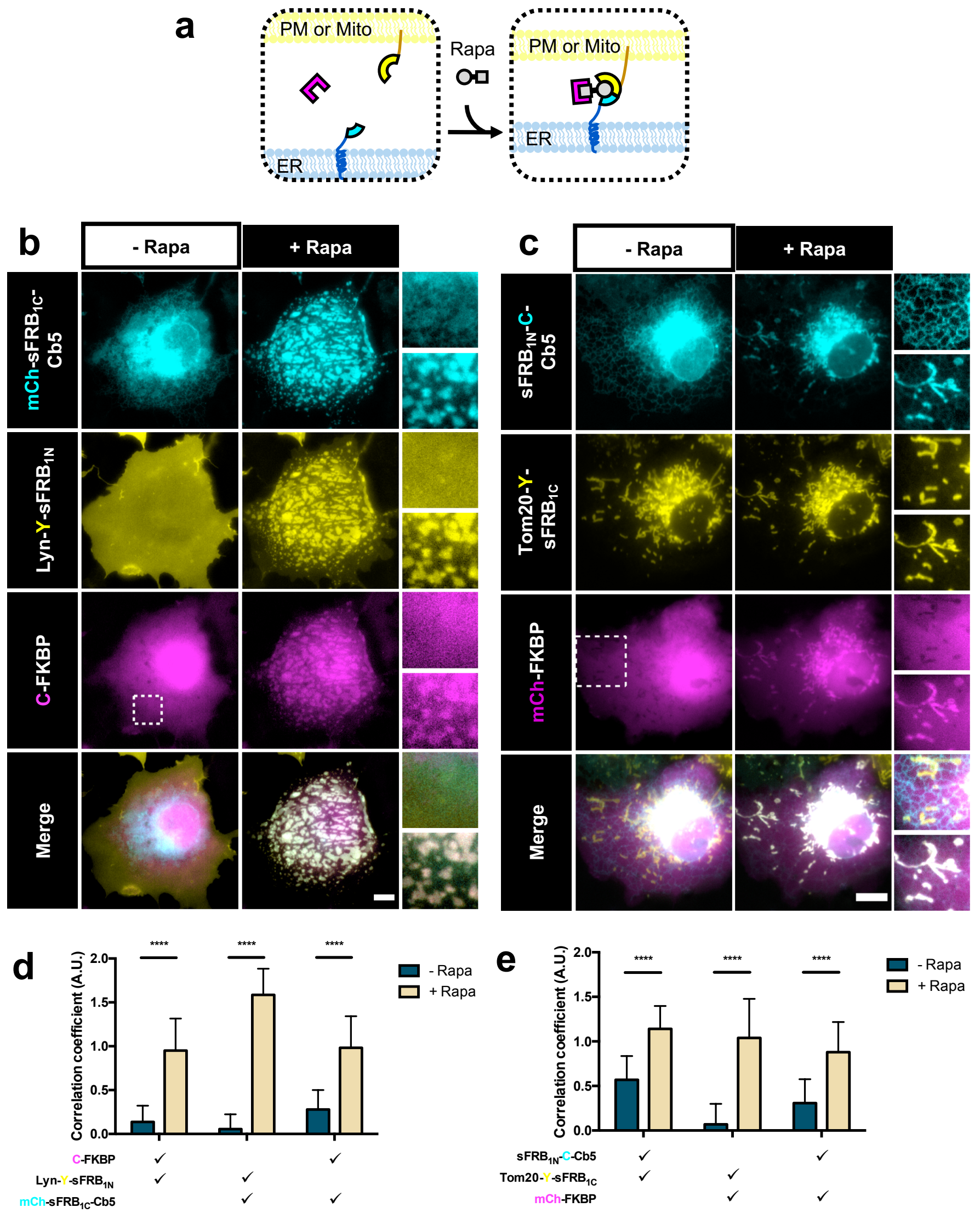
Figure 5
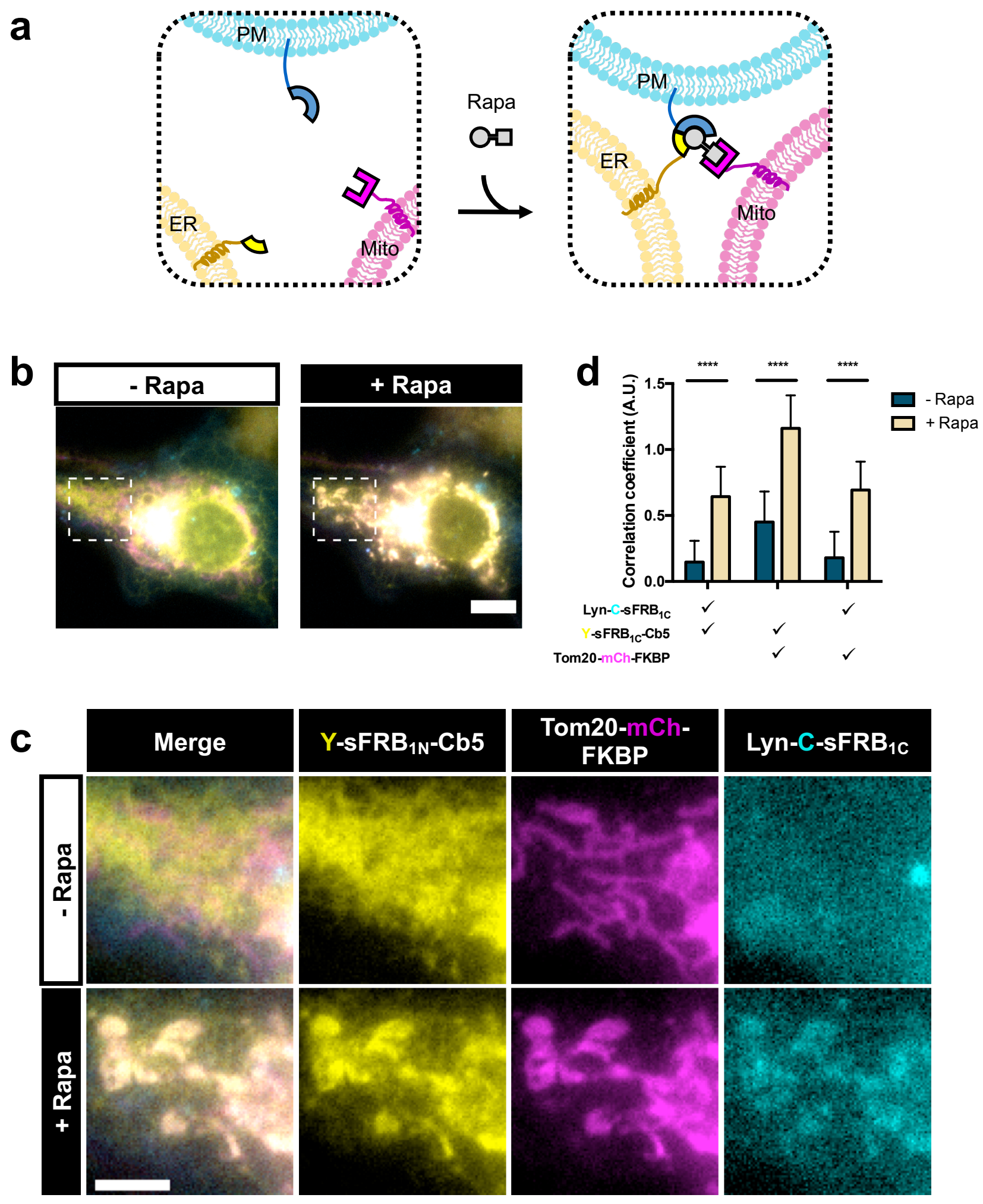

5 um scalebar 
Figure 6
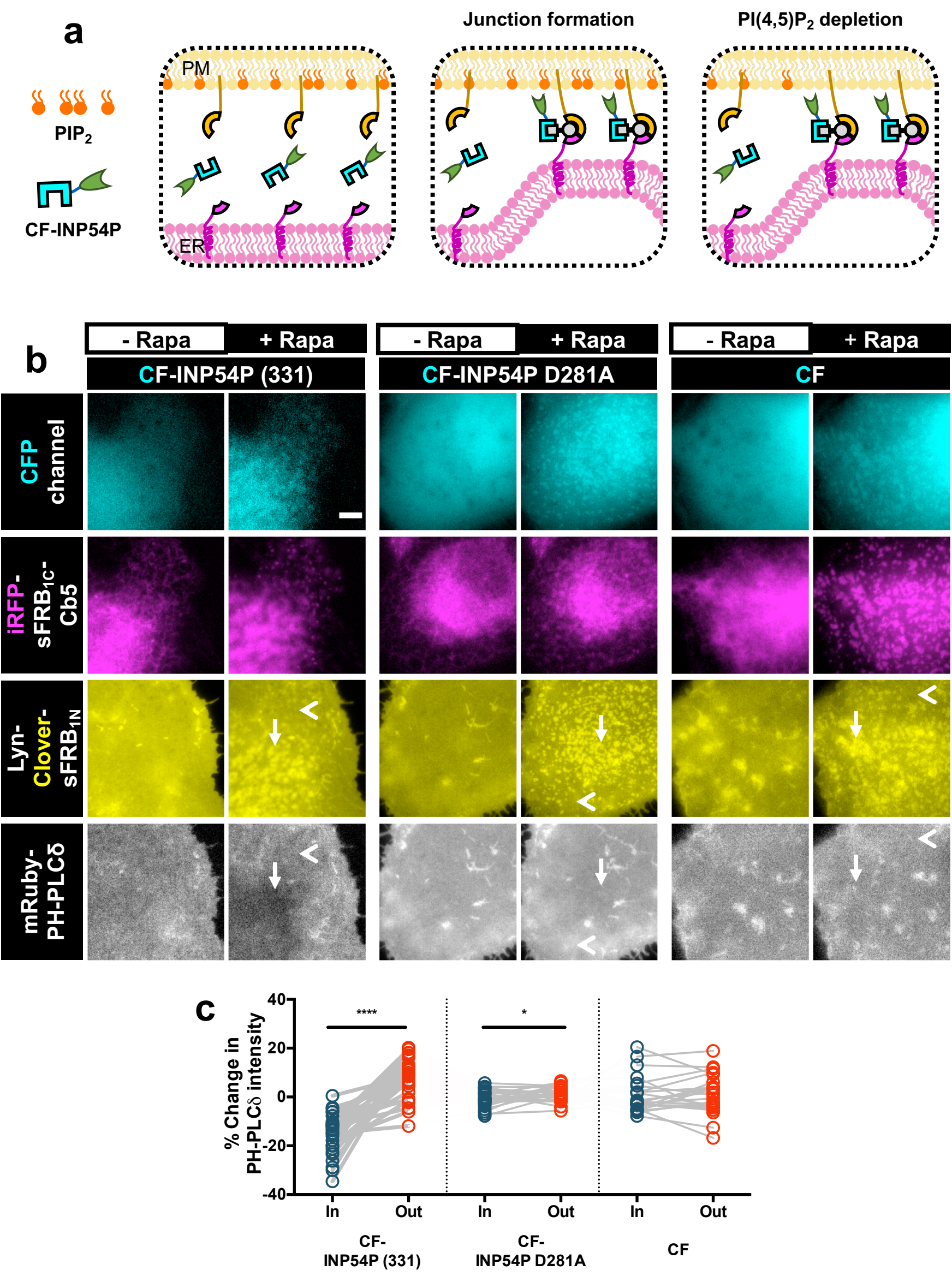